\title{
Wide-Area Assessment of Aperiodic Small Signal Rotor Angle Stability in Real-Time
}

Jóhannsson, Hjörtur; Nielsen, Arne Hejde; Østergaard, Jacob

Published in:

IEEE Transactions on Power Systems

Link to article, DOI:

10.1109/TPWRS.2013.2271193

Publication date:

2013

Link back to DTU Orbit

Citation (APA):

Jóhannsson, H., Nielsen, A. H., \& Østergaard, J. (2013). Wide-Area Assessment of Aperiodic Small Signal Rotor Angle Stability in Real-Time. IEEE Transactions on Power Systems, 28(4), 4545-4557.

https://doi.org/10.1109/TPWRS.2013.2271193

\section{General rights}

Copyright and moral rights for the publications made accessible in the public portal are retained by the authors and/or other copyright owners and it is a condition of accessing publications that users recognise and abide by the legal requirements associated with these rights.

- Users may download and print one copy of any publication from the public portal for the purpose of private study or research.

- You may not further distribute the material or use it for any profit-making activity or commercial gain

- You may freely distribute the URL identifying the publication in the public portal

If you believe that this document breaches copyright please contact us providing details, and we will remove access to the work immediately and investigate your claim 


\title{
Wide-Area Assessment of Aperiodic Small Signal Rotor Angle Stability in Real-Time
}

\author{
Hjörtur Jóhannsson, Member, IEEE, Arne Hejde Nielsen, Senior Member, IEEE, and \\ Jacob Østergaard, Senior Member, IEEE
}

\begin{abstract}
This paper presents the details of a new real-time stability assessment method. The method assesses a particular mechanism of stability: each generator's capability to generate sufficient steady state electromechanical torque. The lack of sufficient steady state torque causes aperiodic increase in rotor angle and a loss of synchronism, referred to as aperiodic small signal instability. The paper provides the theoretical background of the method and an analytical assessment criterion. Furthermore, a mathematical mapping of the generators' operating points that enables informative visualization of multiple operating points is derived in the paper. Finally, results from time-domain simulation of instability scenarios in the Nordic32 test system are presented and results used for testing the assessment method. The results illustrate the method's capability to efficiently identify the location of the emerging problem and to quantify margins to stability boundary.
\end{abstract}

Index Terms-Power system measurements, power system monitoring, power system stability.

\section{INTRODUCTION}

$\mathbf{S}$ ECURITY assessment is a key function in system operation used to identify contingencies that cause stability problems and is traditionally carried out in an offline process. Security assessment in real-time has been impractical due to high computational burden of traditional security assessment methods.

Prior the deregulation of power systems, the system operators knew the most critical contingencies that posed a threat to the stable operation due to their experience of operating the system. The seasonal, weekly, daily and hourly variations in system consumption were well known, and consumption patterns remained nearly unchanged over the years.

The deregulation of power systems resulted in an increased diversity of transactions leading to new consumption and production patterns that added significant complexity to the system operation. Furthermore, the gradually increasing share of power production based on uncontrollable fluctuating renewable energy sources (e.g., wind and solar energy) contribute to the fact

Manuscript received December 31, 2012; revised May 09, 2013; accepted June 17, 2013. Paper no. TPWRS-01416-2012.

H. Jóhannsson is with the Technical University of Denmark, Department of Electrical Engineering, Kgs. Lyngby 2800, Denmark (e-mail: hj@elektro.dtu. $\mathrm{dk})$.

A. H. Nielsen and J. Østergaard are with the Technical University of Denmark, Centre for Electric Technology, Kgs. Lyngby 2800, Denmark (e-mail: ahn@elektro.dtu.dk; joe@elektro.dtu.dk).

Color versions of one or more of the figures in this paper are available online at http://ieeexplore.ieee.org.

Digital Object Identifier 10.1109/TPWRS.2013.2271193 that system operating conditions fluctuate more and, as a consequence of this, the system stability margins also fluctuate more. To cope with such fluctuations, it is desired that the stability margins may be determined in real-time.

Since research and development into this early began, sophisticated computer tools have been developed for use in stability analysis. A general characteristic of these tools is that they can be used to determine whether a given condition is stable or unstable. However, these tools are not efficient in quickly determining stability boundaries or stability margins.

The advances in computational facilities and communications together with the introduction of phasor measurement technology [1] opened up for research and development of real-time applications for wide-area control, protection and monitoring. Overview of some PMU-related applications is provided in few comprehensive surveys [2]-[4]. The applications cover topics such as real-time monitoring and control, improved state estimation, real-time congestion management, adaptive protection and overload monitoring [3].

For real-time stability assessment methods, it is of critical importance that the computational time is sufficiently short. One approach for reducing the computational time for overall system stability assessment is to deploy a set of different assessment methods where each method focuses on the assessment of one particular mechanism of instability. In this way all of the assessment methods can be executed in parallel when a PMU-snapshot is received and the methods' output studied to obtain a view of the overall stability in real-time.

A method that focuses on one particular mechanism of instability has the advantage that the system representation can be simplified, so that the dynamics that have a limited effect on the mechanism of concern can be neglected, thereby reducing the computational burden. In [5], [6] an example of a method aiming at assessing one particular form of instability from phasor measurements is presented. The method utilizes system snapshots for computing sensitivities for the assessment of voltage stability. The method does not relay on a dynamic model to predict the system response, instead basic assumptions and simplifications are applied in the system modeling process resulting in reduced computational burden.

This paper provides the details of a stability assessment method which also focuses on one particular mechanism of stability: the capability of each generator to generate sufficient synchronizing torque so that operation at stable equilibrium point can be maintained. The lack of sufficient steady-state synchronizing torque causes aperiodic increase in rotor angle 


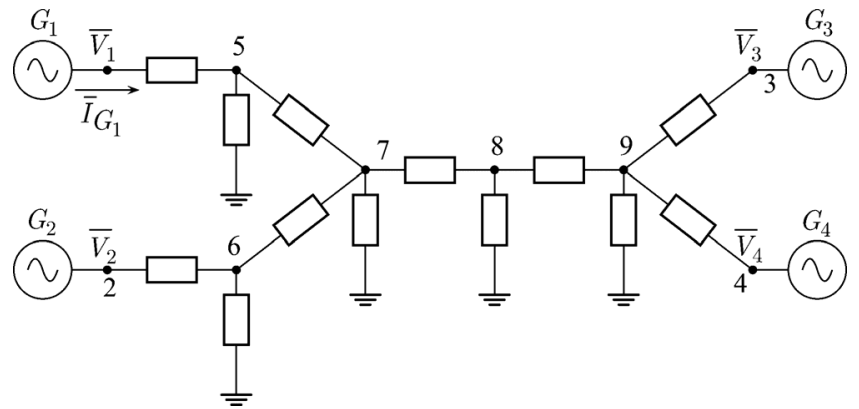

Fig. 1. System used when deriving equations for maximum injectable power. The system loads are represented as impedances and the generators are assumed to maintain constant terminal voltage in the following derivations.

and a loss of synchronism. This form of instability is referred to as aperiodic small signal instability in the following.

The presented method is based on work from a Ph.D. dissertation [7], an overview of the method was provided in [8] and a patent for the method has been granted [9].

\section{ASSESSMENT METHOD}

A method for determining aperiodic small signal stability of individual generators in a power system is presented in the following. As input, the method requires measurement data that provide full observability of the system grid in real-time (e.g., by deploying synchronized wide-area PMU measurements). With full observability of the grid a deterministic representation of the system conditions can be established, where the following simplifying assumptions are made:

- All generators' injections enter the network in a node of constant steady state voltage magnitude:

- The node of constant voltage magnitude is either at the terminal of the machine or behind the saturated direct axis reactance $X_{d}^{s}$ depending on the machine excitation control (manual or automatic) and the status of the machine's over excitation limiter (OEL).

- Loads are represented by their impedance values:

- Longer term load dynamics not included.

- The method evaluates the instantaneous operating conditions, therefore are the instantaneous impedance values as seen from the generators used.

The system conditions are represented by an extended system admittance matrix which includes the network impedances, the generator's direct axis reactances and the load impedances. The representation of power injections at nodes of constant steadystate voltage magnitudes reduces the number of the degrees of freedom associated with the determination of the boundaries for aperiodic small signal stability.

The system in Fig. 1 will be considered when deriving boundaries for aperiodic small signal stability. The figure shows a system where all loads are represented as constant impedances and where all generators are assumed to maintain a constant terminal voltage. With all system impedances known, the system operating conditions can be determined from the generators terminal voltages $\left(\bar{V}_{1}, \bar{V}_{2}, \bar{V}_{3}\right.$ and $\left.\bar{V}_{4}\right)$.

The boundary for aperiodic small signal stability represents the condition when a given generator reaches the maximum power it can inject into a system node in steady state. In the following, the point for maximum injectable power is determined for a given steady state condition described by the impedance network and the four complex voltage phasors $\left(\bar{V}_{1}-\bar{V}_{4}\right)$. Since the voltage magnitude at the nodes of injection is constant, only changes in voltage phase angles at injection buses can result in changes in power injection. The boundary of maximum injectable power for $G_{1}$ is determined by fixing $\bar{V}_{2}, \bar{V}_{3}$ and $\bar{V}_{4}$ and finding the phase angle $\delta_{1}\left(\bar{V}_{1}=\left(V_{1} \angle \delta_{1}\right)\right)$ at which the point of maximum injectable power occurs.

The changes in current injection into node 1 can be determined by applying the superposition principle. The terminal voltage of $G_{1}$ is changed from $V_{1} \angle \delta_{1}$ to $V_{1} \angle \delta_{1}+\Delta \delta$. By superposition, the current from $G_{1}\left(\bar{I}_{G_{1}}\right)$ is expressed as the sum of the current flowing from $G_{1}$ when all other constant voltage nodes shorted $\left(\bar{I}_{\text {sup }, G 1}\right)$ plus the current contributions due to all other constant voltage nodes when node 1 is shorted:

$$
\bar{I}_{G_{1}}=\bar{I}_{s u p, G 1}+\sum_{i=2}^{N} \bar{I}_{s u p, G i} .
$$

$N$ is the number of generators. For a change in $\delta_{1}$, the sum in (1) is unchanged as it is independent of $\bar{V}_{1}$. The current due to $V_{1}$ when all other sources are shorted $\left(\bar{I}_{\text {sup }, G 1}\right)$ does, on the other hand, have the same magnitude but its angle increases by $\Delta \delta$. The apparent power $S_{G 1}$, injected into node 1, becomes

$$
\bar{S}_{G 1}=\bar{V}_{1} \bar{I}_{G_{1}}^{*}=\bar{V}_{1} \bar{I}_{s u p, G 1}^{*}+\bar{V}_{1} \sum_{i=2}^{N} \bar{I}_{s u p, G i}^{*} .
$$

For changes in $\delta_{1}$, the apparent power due the term $\bar{V}_{1} \bar{I}_{\text {sup }, G 1}^{*}$ is constant since the magnitudes and relative angle of $\bar{I}_{s u p, G 1}$ and $\bar{V}_{1}$ is unchanged. The increase in injected power is therefore due to the current component represented by the sum in (2). The point of maximum injectable power from $G_{1}$ occurs therefore when the voltage $\bar{V}_{1}$ is aligned with the current phasor represented by the sum in (2).

By considering Fig. 1 it should be noted that the impedance that $G_{1}$ looks into, when all other points of constant voltage are shorted, is the same as the Thevenin impedance between node 1 and any of the other nodes of constant voltages. Utilizing this, the current $\bar{I}_{G_{1}}$ can be expressed as

$$
\bar{I}_{G_{1}}=\frac{\bar{V}_{1}}{\bar{Z}_{T H}}-\sum_{i=2}^{N} \frac{\bar{V}_{T H, G i}}{\bar{Z}_{T H}}=\frac{\bar{V}_{1}}{\bar{Z}_{T H}}-\frac{\bar{V}_{T H}}{\bar{Z}_{T H}}
$$

where $\bar{V}_{T H}=\sum_{i=2}^{N} \bar{V}_{T H, G i}$ and $\bar{V}_{T H, G i}$ is the Thevenin voltage behind the Thevenin impedance that is used to determine the current due to the voltage source at bus $i$. Utilizing the fact that the maximum injectable power occurs when the current contribution represented by the sum in (1) is aligned with $\bar{V}_{1}$, the maximum injectable power occurs when

$$
\arg \bar{V}_{1}=\arg -\frac{\bar{V}_{T H}}{\bar{Z}_{T H}} .
$$

If $\bar{V}_{T H}$ is used as phase angle reference, the above conditions for the point of maximum injectable power become

$$
\delta_{1}=180^{\circ}-\phi_{T H}
$$




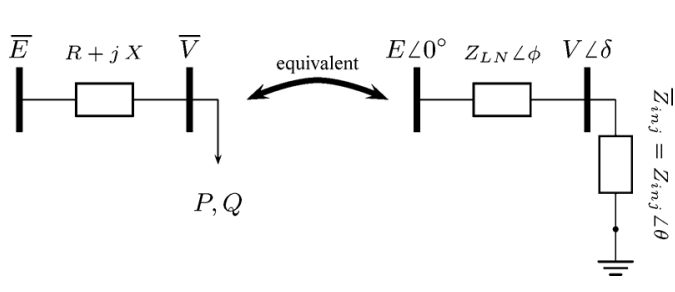

Fig. 2. Two-bus system for which critical and characteristic curves expressed in terms of injection impedance $\bar{Z}_{i n j}$ in [10].

where $\phi_{T H}$ is the angle of $\bar{Z}_{T H}$. The result in (5) can directly be related to results presented in [10] where analytical derivation of the mapping of critical and characteristic curvatures on a three dimensional PQV-surface into the injection plane were derived for the system shown in Fig. 2.

One of the critical curves mapped into the injection impedance plane was the curve satisfying $\partial P / \partial Q=0$, which in fact represents the maximum or minimum injectable power when the voltage magnitudes in both ends $(E$ and $V)$ are fixed. It was shown that the condition where $\partial P / \partial Q=0$ is in fact the same condition as if $\partial V / \partial Q=0$. By equating the expressions for $\partial P / \partial Q=0$ and $\partial V / \partial Q=0$ and solving for $\bar{Z}_{i n j}$ results in the following expression in polar form:

$$
Z_{i n j}=-\frac{Z_{L N} \sin \theta}{\sin \phi}
$$

where $\theta$ is the angle of the injection impedance $\bar{Z}_{i n j}$. Analytical expressions for lines of constant $V, P, Q$ and $\delta$ were also derived in [10]. The curve of constant phase angle difference $\delta$ between $\bar{E}$ and $\bar{V}$ becomes

$$
Z_{i n j}=-\frac{Z_{L N} \sin (\delta+\phi-\theta)}{\sin \delta} .
$$

The curve of constant phase angle difference where $\delta=$ $180^{\circ}-\phi$ in (7) is in fact the same as the curve representing the conditions $\partial P / \partial Q=0$ and $\partial V / \partial Q=0$ as given by (6).

The obtained expression in (5) means that the boundary represented in [10], derived for a simple two-bus system, can be applied to an arbitrary system. Hence, the boundaries of a given machine can be determined if the Thevenin impedance, seen from the machine's node of constant steady state voltage magnitude, and the corresponding injection impedance are known. The stability boundary of a given generator given by (5) can therefore be expressed as

$$
Z_{i n j, i}=-\frac{Z_{T H, i} \sin \theta}{\sin \phi_{T H, i}} .
$$

Fig. 3 shows how the stability boundary for aperiodic small signal stability, given by (8), appears in the injection impedance plane. The boundary appears as a circle with radius $r=Z_{T H} /\left(2 \sin \phi_{T H}\right)$. An operation outside the circle indicates a stable operation where a relative increase in the phase angle at the bus of injection gives increased active power injection. An operation inside the circle represents unstable

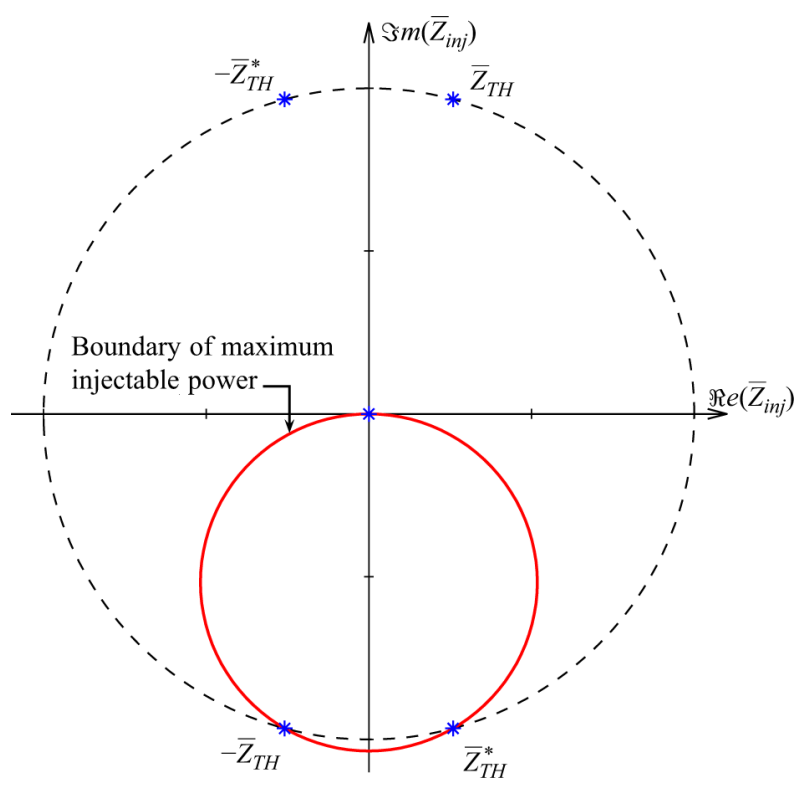

Fig. 3. Boundary of aperiodic small signal stability (red circle) in the injection impedance plane. The boundary is described in terms of the system Thevenin impedance by (8).

conditions characterized a decrease in the injected power as the phase angle increases at the bus of injection. By utilizing (8), the aperiodic small signal stability of a given generator can therefore be described by the following set of inequalities:

$$
\begin{aligned}
&\left|\frac{\bar{Z}_{i n j} \cdot\left(2 \sin \phi_{T H}\right)+j \cdot Z_{T H}}{Z_{T H}}\right| \\
& \times\left\{\begin{array}{l}
>1 \text { Stable } \\
=1 \text { At the boundary } \\
<1 \text { Unstable. }
\end{array}\right.
\end{aligned}
$$

Based on the above analysis, a method for assessing the aperiodic small signal stability of the system generators can be outlined consisting of the steps shown as algorithm 1.

Algorithm 1 Assessment of Aperiodic Small Signal Stability

Input Continuous sequence of system PMU-snapshots

foreach System Snapshot do

foreach Generator $j=1: K$ do

Determine the injection impedance $\bar{Z}_{i n j, j}$

Determine the Thevenin impedance $\bar{Z}_{T H, j}$

Apply (9) to assess the stability

end

end

In recent research [11] the computation of Thevenin impedances for 1325 generators in a 7917-bus system takes $2.5 \mathrm{~ms}$, which makes real-time assessment feasible. 


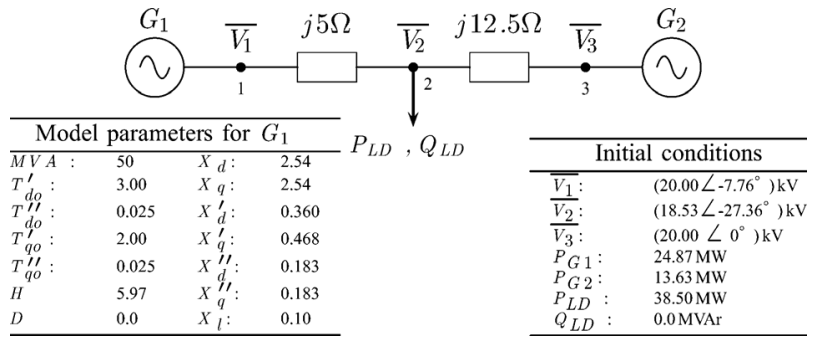

Fig. 4. Three-bus system from [8] used to test the accuracy of the assessment method. The system voltage is $20 \mathrm{kV}$ line-to-line and the generator $G_{3}$ represents an infinite bus $(H \rightarrow \infty)$. Generator $G_{1}$ is manually excited and is operated with constant mechanical power input.

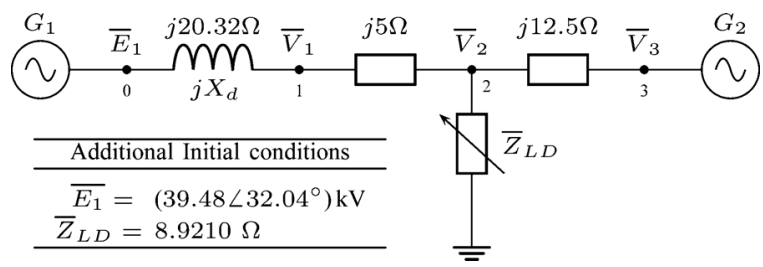

Fig. 5. Extended system representation of the system in Fig. 4. Power injections are represented at nodes of constant steady state voltage magnitude and the load at bus 2 is represented by its impedance $\bar{Z}_{L D}$.

\section{Simple Example-Test of the Method's ACCURACY TO DETECT INSTABILITY}

In [8] a time domain simulation was used to verify the method's capability to detect instability. An aperiodic rotor angle instability of a three bus system was simulated and the simulation output used to create PMU snapshots that were used as input to the assessment method for testing purposes.

In the following, the same test scenario is revisited. The focus is on investigating how accurately the method can detect instability. Fig. 4 shows the system from [8] which is used in the following. The system consists of three $20 \mathrm{kV}$ buses, two generator buses and one load bus. Generator $G_{2}$ at bus 3 represents an infinite bus where the voltage at bus 3 is constant during the analysis. Generator $G_{1}$ is a 50 MVA round rotor machine that is manually excited and operated with a constant mechanical power input. The dynamic model parameters for $G_{1}$ are provided in Fig. 4 together with initial conditions, with purely resistive load of $38.5 \mathrm{MW}$ and production of $24.87 \mathrm{MW}$ from $G_{1}$. In the following, the loss of synchronous operation of $G_{1}$ is provoked by applying two minor increase in load demand on bus 2 .

\section{A. Analysis of the Initial Condition}

The detection method is based on that the power injections from the system generators are represented at nodes of constant steady state magnitude. For the system in Fig. 4, the injections from generator $G_{1}$ are represented by a source of constant voltage magnitude behind $X_{d}$, the voltage at the infinite bus is assumed to be constant, and the load at bus 2 is represented by its impedance as illustrated in Fig. 5.

Since the steady state voltage magnitudes at the points of injections are constant, all potential operating conditions (for the chosen manual excitation of the machine) can be determined from the value of the phase angle difference between the two nodes of constant voltage $(\Delta \delta)$ and the value of load impedance

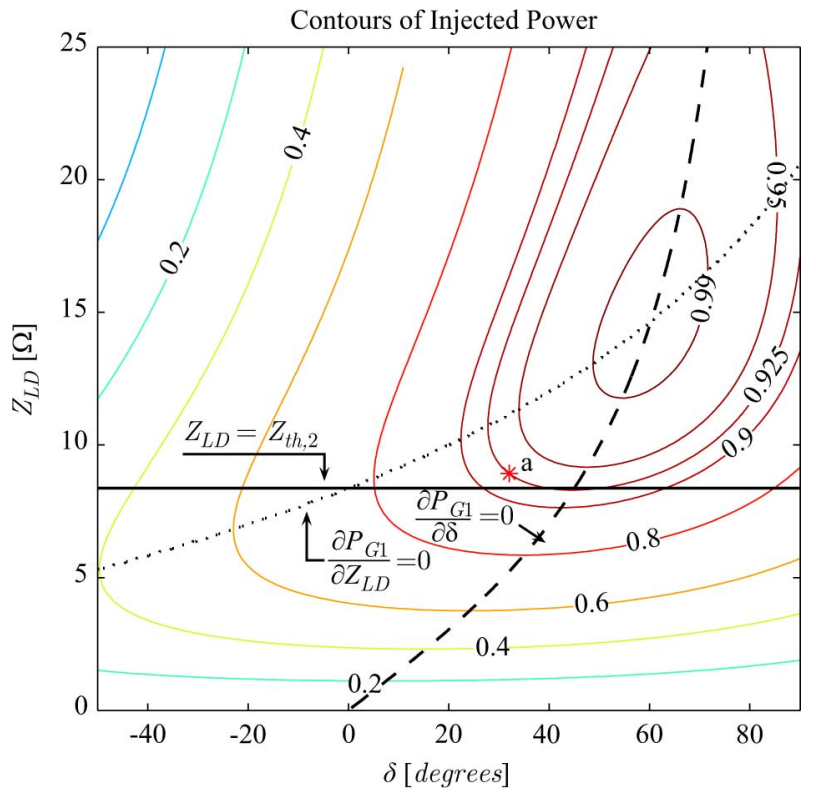

Fig. 6. Contours of constant injected power in the $\delta-Z_{L D}$ plane for $G_{1}$ in Fig. 4 . The dashed line represents the boundary of maximum injectable power for a fixed $Z_{L D}$ and the dotted line represents maximum injectable power for a fixed $\delta$. The contours show per unit values where the maximum injectable power is used as base value.

$\left(\overline{Z_{L D}}=R_{L D}+j X_{L D}\right)$. That is, the constrained system only has three degrees of freedom $\left(\Delta \delta, R_{L D}\right.$ and $\left.X_{L D}\right)$. If the load impedance is constrained to a fixed load angle, the system operating point can be described in terms of $\Delta \delta$ and the load impedance magnitude $Z_{L D}$.

In the following analysis, the load power factor is kept at unity and the power injection from $G_{1}$ into the node of constant voltage (behind $X_{d}$ ) is inspected with respect to variations of $\Delta \delta$ and $Z_{L D}$. Fig. 6 shows how the power injection from $G_{1}$ varies as a function of these two variables. The infinite bus is used as an angle reference resulting in $\Delta \delta=\delta$ where $\delta$ is the angle of the voltage behind $X_{d}$.

The figure shows contours of constant injected power in the $Z_{L D}-\delta$ plane. The contours have been normalized in such a way, that the value of maximum injectable power is used as per unit base. The initial conditions, from Fig. 4, are represented at the point $a$ that is on the contour where $P=0.925$ pu (equivalent to $P_{G 1}=24.87 \mathrm{MW}$ ). The figure contains three characteristic lines; a dotted line representing the conditions where the maximum injectable power for a fixed value of delta occur $\left(\partial P_{G 1} / \partial Z_{L D}=0\right)$, a dashed line where the maximum amount of injectable power for a fixed value of $Z_{L D}$ occur $\left(\partial P_{G 1} / \partial \delta=\right.$ 0 ) and a straight line representing where the load impedance $Z_{L D}$ equals the magnitude of system Thevenin impedance, seen from the load at bus $2\left(Z_{t h, 2}\right)$.

In this study, the dashed line (where $\partial P_{G 1} / \partial \delta=0$ ) is of interest. The line represents the boundary of aperiodic small signal stability for machine $G_{1}$. An operation in the region to the right of the dashed line, for fixed $Z_{L D}$, is characterized by that an increase in $\delta$ causes a reduced injection of power. This means that a small increase of $\delta$ would lead to acceleration of the machine causing further increase in the angle $\delta$. Since the machine acceleration increases with increasing $\delta$, an operation to the right 


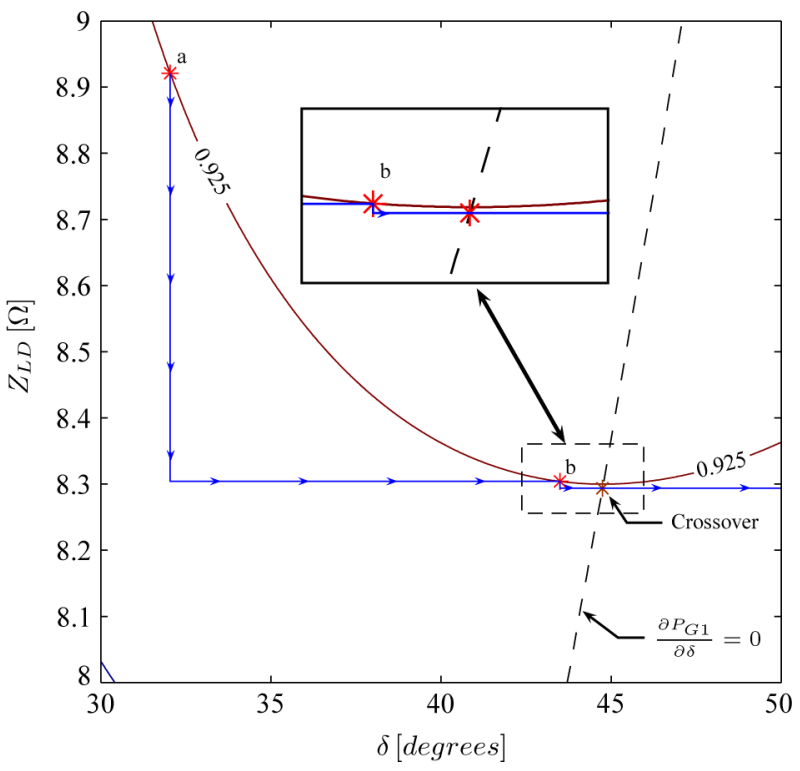

Fig. 7. Enlarged view of Fig. 6. Points $a$ and $b$ are the initial equilibrium and the new equilibrium after the first disturbance, respectively. After the second disturbance, no intersection is between the contour of constant $P_{G 1}$ and the new value for $Z_{L D}$, hence $G_{1}$ loses synchronism.

of the dashed line is unstable and would gradually cause $G_{1}$ to lose synchronism with the infinite bus.

In the example, the machine $G_{1}$ is operated with a constant mechanical power input. This means that the contour $P=0.925 p u$ (in Fig. 6) represents the trace of possible steady state operating points for changes in $Z_{L D}$. If the initial conditions are considered (point $a$ in Fig. 6), then a small decrease in the load impedance $Z_{L D}$ (corresponds to increased load demand) results in a new steady state point, located on the contour $P=0.925 p u$, but closer to the boundary.

In the following time domain simulation, a loss of aperiodic small signal stability of $G_{1}$ is provoked by applying two small step decreases in the load impedance $Z_{L D}$. The effect of the two disturbances is illustrated in Fig. 7, which shows an enlarged view of the contour plot in Fig. 6. In the figure, point $a$ represents the initial operating conditions. The first disturbance to be applied is a decrease in $Z_{L D}$ from $8.9210 \Omega$ to $8.3039 \Omega$ which results in $G_{1}$ approaching a new equilibrium represented by the point $b$. The post-disturbance equilibrium at point $b$ is stable, but very close to the stability boundary. The second disturbance applied, is the very small decrease in $Z_{L D}$ from $8.3039 \Omega$ to $8.2936 \Omega$ which results in the loss of aperiodic small signal stability of machine $G_{1}$. The effect of the disturbance can be seen by considering the box containing an enlarged view of the region close to point $b$. It can be seen that the contour of constant $P_{G 1}$ and the line representing the new value of $Z_{L D}$ do not intersect after the second disturbance. As a result, no equilibrium can be obtained, and consequently the machine eventually loses synchronism.

\section{B. Simulation Results}

The time domain simulation was carried out in PSS/E (version 30). The initial conditions from Fig. 8 and the two previously mentioned load increases were applied to provoke an ape-
Load Impedance on bus $2\left(Z_{L D}[\Omega]\right)$
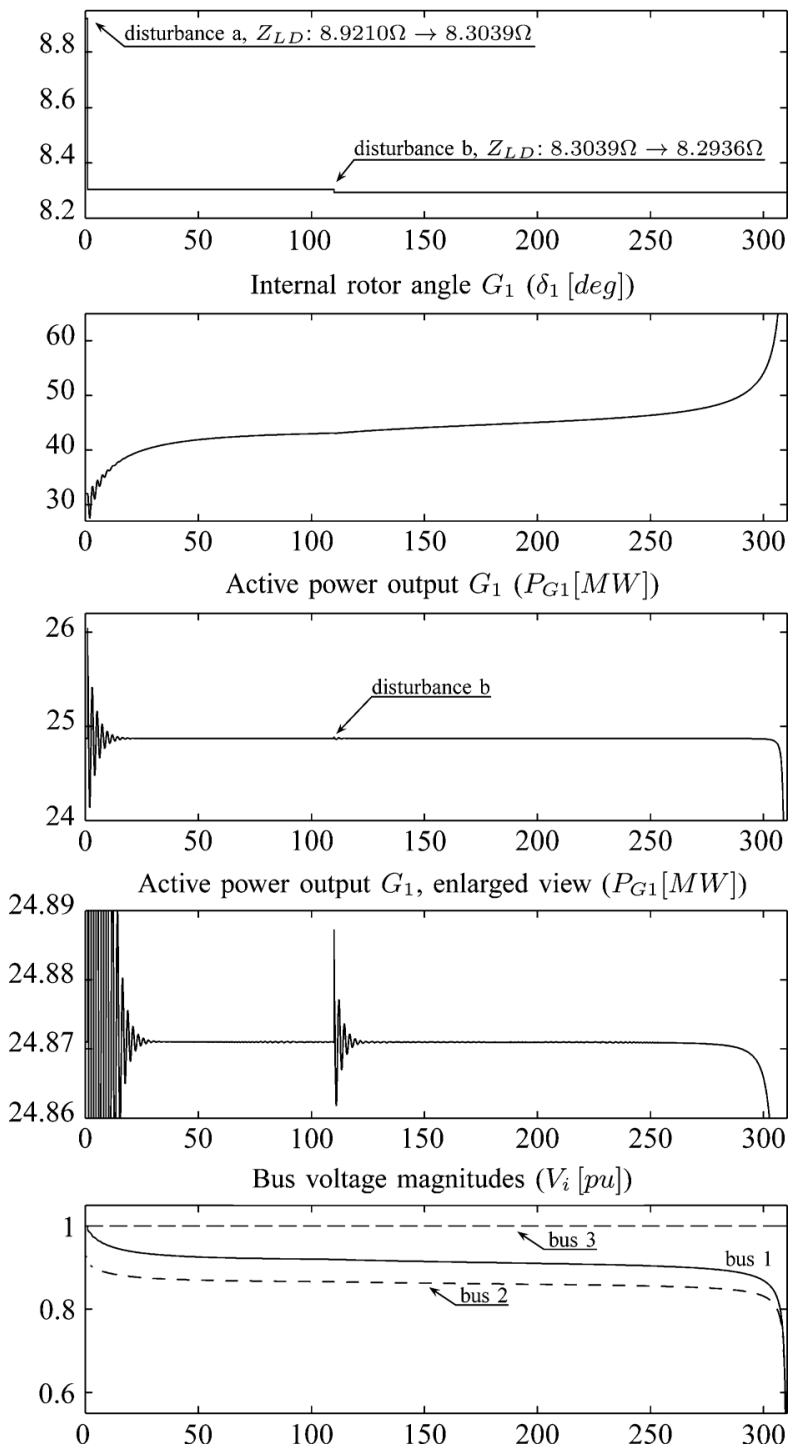

Fig. 8. Simulation results. The first plot shows the two applied disturbances and below are plots of the internal rotor angle and active power output from machine $G_{1}$ and the bus voltage magnitudes.

riodic small signal instability. The results from the time domain simulation are shown in Fig. 8. The first plot shows the applied changes in the load impedance that eventually caused the system instability. The first disturbance was applied at $t=1 \mathrm{~s}$ where the load impedance was changed from $8.9210 \Omega$ to $8.3039 \Omega$. The second disturbance was applied at the time $t=110 \mathrm{~s}$ where the impedance was changed from $8.3039 \Omega$ to $8.2936 \Omega$. It can be seen from the other plots that approximately $200 \mathrm{~s}$ passed from the second disturbance until a very sudden decrease in the system voltages occurred.

The second plot from top shows the internal rotor angle of $G_{1}$, where the infinite bus is used as the angle reference. After the second disturbance $(t=110 \mathrm{~s})$, a very slow increase in the rotor angle was experienced over a period of approximately $200 \mathrm{~s}$ before a very sharp increase in the rotor angle occurred.

The third and the fourth plot show the power output from $G_{1}$ $\left(P_{G 1}\right)$. The fourth plot provides an enlarged view of the third plot. The second disturbance can barely be seen in the third 
plot, despite this it is still sufficient to cause $G_{1}$ to lose its synchronous operation. In the fourth plot, the size of the second disturbance can be seen more clearly. It is worth noticing, that the power output from $G_{1}$ was constant following the second disturbance until the sudden sharp decrease in the power output occurred. The emerging stability problems of $G_{1}$ are not reflected in the power output from the machine and could therefore not have been identified from observations $P_{G 1}$ alone.

The bottom plot in Fig. 8, shows the bus voltage magnitudes in the system. Following the first disturbance, the bus voltages on buses 1 and 2 appear to decrease very slowly until a very fast decrease occurs.

\section{Test of the Assessment Method}

The time domain simulation output was used to generate synthetic PMU-measurements of voltage and current phasors every $20 \mathrm{~ms}$ of the simulated time interval. To test the assessment method, the snapshots were used to determine the injection impedance for $G_{1}\left(\bar{Z}_{i n j}\right)$ and the system line and load impedances. With all network impedances known, the system Thevenin impedance $\bar{Z}_{T H}$, as seen from the point of injection, could be determined. The value of $\bar{Z}_{i n j}$ and $\bar{Z}_{T H}$ was determined for every PMU-snapshot and (8) deployed to determine when the stability boundary was crossed.

Fig. 9 depicts the test results. The figure shows four plots, where in (a) a time domain plot of the voltage magnitude at bus 2 is shown. The dots on the plotted line are used to illustrate a fixed interval of time where the distance between two adjacent dots represents $\Delta t=5 \mathrm{~s}$. Three events are marked on the plots, where points $a$ and $b$ denote the time when the two disturbances were applied, and point $c$ is used to denote when the stability boundary was crossed. The time of detected instability occurred approximately $71 \mathrm{~s}$ after the second disturbance and it is worth noticing that approximately $130 \mathrm{~s}$ elapsed from the detection of the boundary crossover until the very sharp decline in voltage magnitude occurred.

Plots (b)-(d) in Fig. 9 all show the same trajectory of the injection impedance $\bar{Z}_{i n j}$, but with different levels of detail. The value of the injection impedance $\bar{Z}_{i n j}$ is normalized in such a way that the stability boundary, at any instant of time, always appears as a circle with a diameter at unity. In plot (b), the trajectory of the injection impedance can be seen for nearly the whole time interval shown in (a). It can be seen that the initial conditions (point $a$ ) are quite close to the stability boundary, and when the disturbances are applied the trajectory slowly moves towards the boundary. The boundary is crossed at point $c$ after which the machine gradually accelerates and loses synchronism with the infinite bus.

Plot (c) shows an enlarged view of the trajectory from $a$ through $b$ to $c$. It can be seen that the system gradually approaches the operating point $b$ following the first disturbance where the distance between two dots (which denotes a time interval of $5 s$ ) gradually decreases. A gradual decrease in the distance between two points indicates that the machine is gradually decelerating and approaching an equilibrium point.

Plot (d), provides an even more enlarged view of the injection impedance trajectory from the occurrence of the second, very small disturbance, and until after the boundary is crossed. If the trajectory from $b$ to $c$ is studied, it can be seen that the disturbance initially caused increase in the average angular velocity of the machine, which is reflected by a gradual movement of the system operating point towards the boundary. As the operating point moves closer to the boundary (from $b$ to $c$ ), the distance between adjacent dots gradually decreases, which indicates that machine is slowly decelerating towards synchronous speed during that period. When the boundary is crossed, it can be seen that the distance between the dots gradually increases. This indicates a machine is acceleration, which is expected behavior when the boundary for aperiodic small signal instability has been crossed.

The test result in Fig. 9 show that, for the studied system, the suggested assessment method accurately detects when the boundary of aperiodic small signal stability is crossed.

Furthermore, it is interesting to see how long it can take from the initial crossing of the stability boundary until the sharp decline in the voltage magnitude occurs. During this time interval the machine is slowly but gradually accelerating, yet it is very hard to identify that the machine was unstable merely by inspecting the plots of the system voltage magnitude and power output in Fig. 8.

\section{INFORMATIVE VISUALIZATION of Multiple Operating PoINTS}

\section{A. Margins to the Stability Boundary}

The presented assessment method enables monitoring of a stability boundary of a given generator. For real-time assessment the margin from an observed operating point to its stability boundary might be an useful early warning indicator. The stability boundary related to a given operating point $\left\{\bar{Z}_{i n j, i}, \bar{Z}_{t h, i}\right\}$, is represented by a circle in the injection impedance plane. However, a margin from the point $\bar{Z}_{i n j, i}$ to its stability boundary expressed in terms of injection impedance does not provide a useful physical interpretation of the distance to instability. In order for a physical interpretation of the margins to be useful, it is preferable to express margins in quantities such as active power or even as a percentage active power margin to the maximum injectable power.

The stability boundary is represented by the line of constant phase angle where $\delta=180-\phi_{t h}$ when the power injection is represented at a node of constant voltage magnitude. Due to the algebraic expression for the stability boundaries, the margin from a observed operating point $\left\{\bar{Z}_{i n j, i}, \bar{Z}_{t h, i}\right\}$ to its corresponding boundaries can be expressed in terms of several different variables. For the purpose of deriving an active power margin, the apparent power injection is expressed in terms of Thevenin voltage and the Thevenin impedance:

$$
\begin{aligned}
\bar{S}_{i n j} & =\left(\frac{\bar{E}_{t h}-\bar{V}}{\bar{Z}_{t h}}\right)^{\star} \bar{V} \\
& =\frac{E_{t h} V}{Z_{t h}} e^{j\left(\delta+\phi_{t h}\right)}-\frac{V^{2}}{Z_{t h}} e^{j\left(\phi_{t h}\right)}
\end{aligned}
$$

where $\bar{E}_{t h}$ is used as a phase angle reference. The active power injection becomes

$$
P_{i n j}=\frac{E_{t h} V}{Z_{t h}} \cos \left(\delta+\phi_{t h}\right)-\frac{V^{2}}{Z_{t h}} \cos \left(\phi_{t h}\right) .
$$


Voltage Magnitude at Bus 2

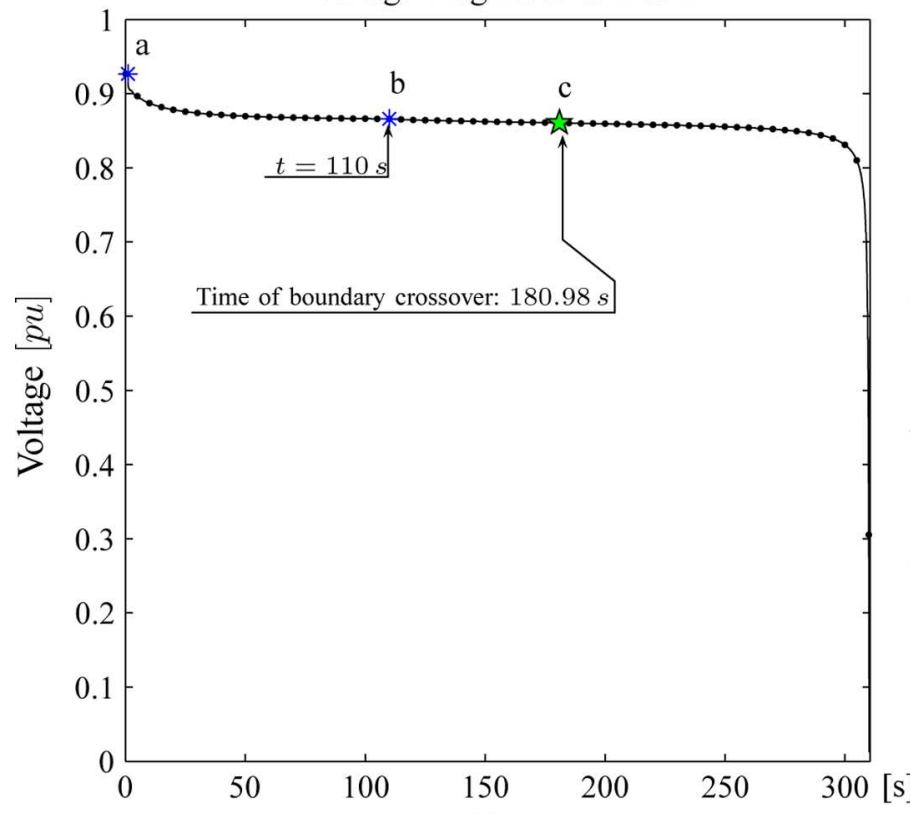

(a)

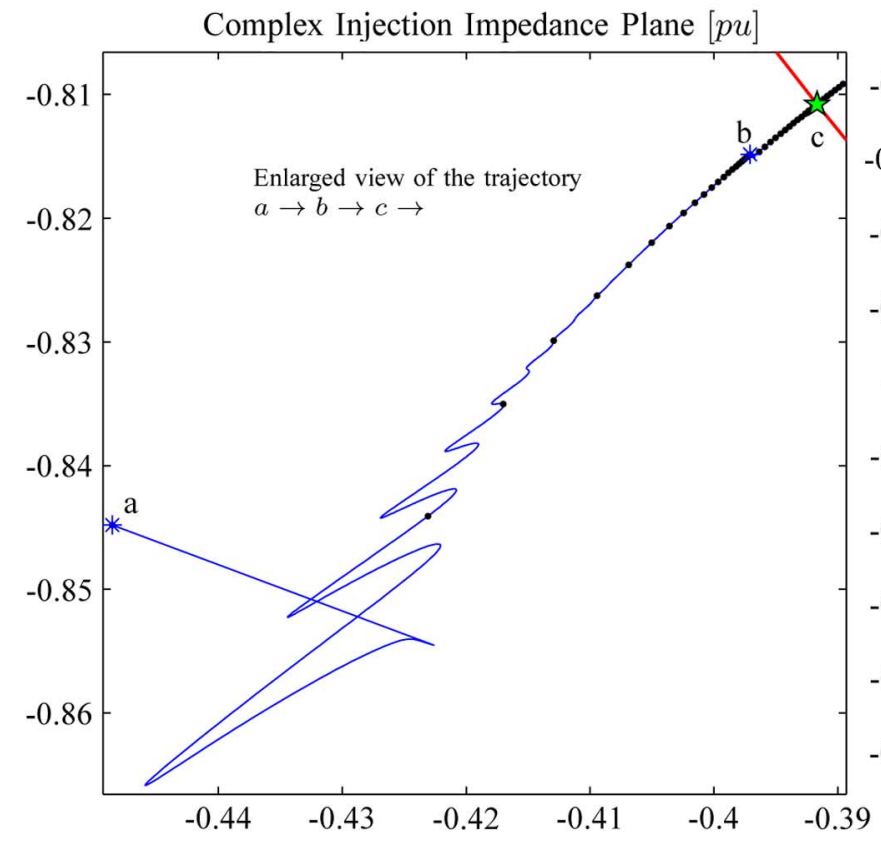

(c)

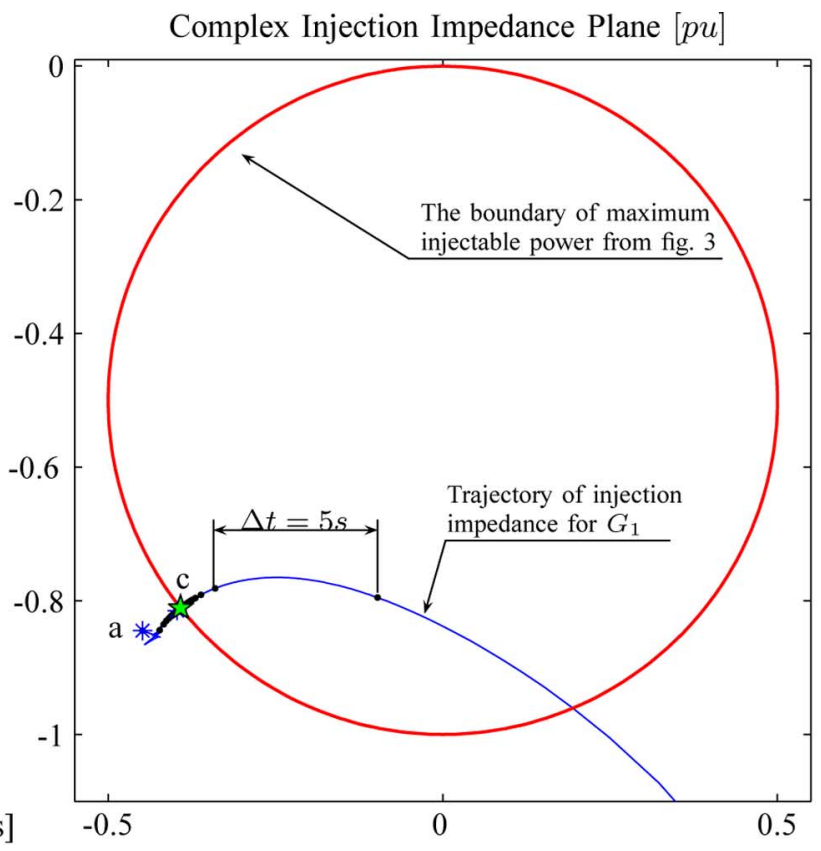

(b)

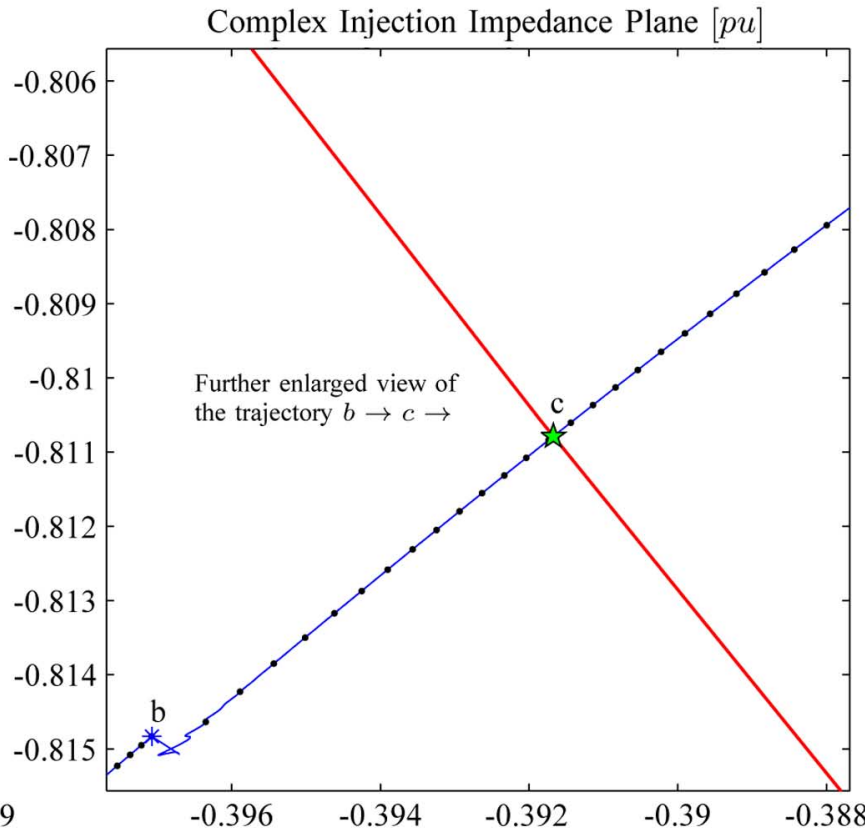

(d)

Fig. 9. Test results. (a) plot of the voltage magnitude on bus 2 where the two disturbances are marked with $a$ and $b$ respectively, and the time when the boundaries are crossed is marked $c$. (b)-(d) the corresponding trajectory of the injection impedance for generator $G_{1}$. The injection impedance values are normalized in such way that the stability boundary has a diameter at unity at any instance of time.

A power injection is represented by a negative value of $P_{i n j}$. The maximum injected power occurs when $\delta=180^{\circ}-\phi_{t h}$ and can be expressed as

$$
P_{i n j, \text { max }}=-\frac{E_{t h} V}{Z_{t h}}-\frac{V^{2}}{Z_{t h}} \cos \left(\phi_{t h}\right) .
$$

An active power margin $\Delta P_{\text {inj }}$ becomes

$$
\begin{aligned}
\Delta P_{i n j} & =P_{i n j, \max }-P_{i n j} \\
& =-\frac{E_{t h} V}{Z_{t h}}\left(\cos \left(\delta+\phi_{t h}\right)+1\right) .
\end{aligned}
$$

A percentage margin $\% \Delta P_{i n j}$ to the maximum injectable power becomes

$$
\begin{aligned}
\% \Delta P_{i n j} & =\frac{\Delta P_{i n j}}{P_{i n j, \max }} \cdot 100 \% \\
& =\frac{\cos \left(\delta+\phi_{t h}\right)+1}{1+\left|\frac{\bar{Z}_{i n j}}{\bar{Z}_{t h}+\bar{Z}_{i n j}}\right| \cos \left(\phi_{t h}\right)} \cdot 100 \% .
\end{aligned}
$$

The above margins describe how much the active power injection can be increased as the phase angle $\delta$ is increased to its 
critical value at $\delta=180-\phi_{t h}$, while other system variables remain fixed. This differs from how stability margins are derived by means of continuation methods [12] where the system is stressed in a particular direction by applying predefined loading and dispatch patterns. These stress patterns are usually based on operational experience, where the daily consumption patterns are known by the system operators.

Even though the stability margins suggested above, that are derived from the phase angle margin $\Delta \delta$, are not obtained by applying specific "normal" stress patterns they do provide useful information. The method is intended to provide a stability assessment during emergency operating conditions. During such conditions, it is not likely that the normal stress patterns will give the most likely stress direction of the system, since many other control and load restoration mechanisms play a more significant role in such situations. The actual stress direction might be dominated by the actions of ULTC-transformers and other devices that try to restore the pre-disturbance consumption. This means that the "normal" stress direction is not suitable for determining a margin to the system stability boundary. On the other hand, the element-wise margins (13)-(14) provide a valuable information concerning which of the system generators is operating close to, or approaching its stability boundaries. Such information could be used to take remedial actions to avoid an emerging instability.

\section{B. Visualization of Multiple Operating Points}

The suggested method performs an element-wise assessment of the generators' aperiodic small signal stability. In a system with $k$ generators, $k$ individual Thevenin impedances $\bar{Z}_{t h, i}(i=$ $1, \ldots, k)$ are determined leading to use of $k$ different stability boundaries for the assessment. In [10] it was shown that the stability boundaries are circular, and therefore it is possible to normalize all the $k$ boundaries in such way that they appear as a circle with radius at unity centered at the origin of the normalized impedance plane. By doing so, all of the $k$ operating points for the generators can be visualized in the same normalized injection impedance plane where all of the points are held against the same stability boundary.

In [10] it was shown how several characteristic lines of constant $P, Q, V$ and $\delta$ can be represented in the injection impedance plane, if only the system Thevenin impedance is known. It would be desirable if some characteristic lines could be derived in a normalized injection impedance plane, so that the lines provide useful information regarding the operating conditions of all normalized operating points.

In the following, it will be shown how a given operating point $\left\{\bar{Z}_{i n j, i}, \bar{Z}_{t h, i}\right\}$ can be mapped into a normalized injection impedance plane so that the voltage phase angle margin $\Delta \delta$ to the critical stability boundary and the lines of constant $V / E_{t h, i}$ ratio are preserved.

1) Mapping of Lines of Constant $V$ and $\Delta \delta$ : In this section, the lines of constant phase angle margin $\Delta \delta$ and normalized voltage $V / E_{t h}$ in the injection impedance plane for an arbitrary $\bar{Z}_{t h}$ will be mapped into a plane where the boundaries are derived from Thevenin impedance that has the same magnitude as $\bar{Z}_{t h}$ and is purely inductive. That is, the operating point

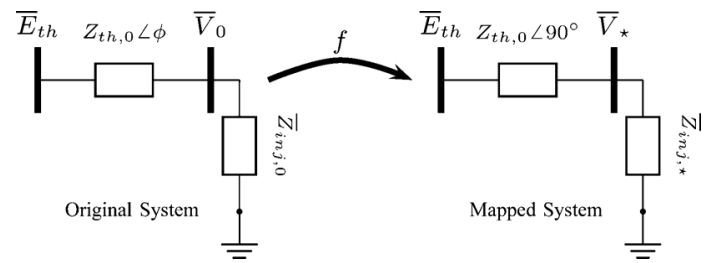

Fig. 10. Mapping of a generator's operating point $\left\{\bar{Z}_{i n j, 0}, \bar{Z}_{t h, 0}\right\}$ to an equivalent operating point $\left\{\bar{Z}_{i n j, \star},\left(Z_{t h, 0} \angle 90^{\circ}\right)\right\}$, such that the normalized voltage magnitude $\left(V_{0} / E_{t h}=V_{\star} / E_{t h}\right)$ and the phase angle margin $\Delta \delta$ are preserved after the mapping.

$\left\{\bar{Z}_{i n j, 0}, \bar{Z}_{t h, 0}\right\}$ is mapped as the point $\left\{\bar{Z}_{i n j, \star},\left(Z_{t h, 0} \angle 90^{\circ}\right)\right\}$ in the injection impedance plane as illustrated in Fig. 10.

In the following, the mapping

$$
f:\left\{\bar{Z}_{i n j, 0}, \bar{Z}_{t h, 0}\right\} \rightarrow\left\{\bar{Z}_{i n j, \star},\left(Z_{t h, 0} \angle 90^{\circ}\right)\right\}
$$

is determined where the following properties of the mapped operating point are preserved:

- The normalized voltage magnitude $V / E_{t h}$ is the same for both operating points.

- The phase angle margin $\Delta \delta$ to the critical boundary is preserved after the mapping.

In the subsequent derivations, $\bar{Z}_{i n j, 0}, \bar{Z}_{t h, 0}, \bar{V}_{0}$ and $\delta_{0}$ are used to denote original variables while $\bar{Z}_{i n j, \star}, \bar{Z}_{t h, \star}, \bar{V}_{\star}$ and $\delta_{\star}$ denote the mapped variables. It is desired that the phase angle difference from operating point to the boundary is preserved after the mapping which results in

$$
\Delta \delta=180^{\circ}-\phi-\delta_{0}=90^{\circ}-\delta_{\star} .
$$

It is also desirable that the normalized voltage magnitude is the same. To achieve this, the following relation between the original voltage phasor $\bar{V}_{0}$ and the mapped voltage phasor $\bar{V}_{\star}$ can be utilized:

$$
\frac{\bar{V}_{\star}}{E_{t h}}=\frac{\bar{V}_{0} e^{j\left(\delta_{\star}-\delta_{0}\right)}}{E_{t h}}=\frac{\bar{V}_{0} e^{j\left(\phi-90^{\circ}\right)}}{E_{t h}} .
$$

The normalized voltage magnitude can be expressed in terms of variables shown in Fig. 10:

$$
\frac{\bar{V}_{\star}}{E_{t h}}=\frac{\bar{Z}_{i n j, \star}}{Z_{t h, 0} e^{j 90^{\circ}}+\bar{Z}_{i n j, \star}}=\frac{\bar{Z}_{i n j, 0}}{\bar{Z}_{t h, 0}+\bar{Z}_{i n j, 0}} e^{j\left(\phi-90^{\circ}\right)} .
$$

Utilizing that $Z_{t h, 0} e^{j 90^{\circ}} e^{j\left(\phi-90^{\circ}\right)}=\bar{Z}_{t h, 0}$ and solving for $\bar{Z}_{i n j, \star}$ gives

$$
\bar{Z}_{i n j, \star}=\frac{1}{\frac{1}{\bar{Z}_{i n j, 0}}+\frac{1}{\bar{Z}_{t h, 0}}\left(1-e^{j\left(\phi-90^{\circ}\right)}\right)} .
$$

Equation (19) describes the mapping in (15) that preserves the above mentioned characteristics.

2) Characteristics of the Normalized Injection Impedance Plane: In order to normalize the stability boundary for aperiodic small signal stability, it is relevant to consider how the boundary appears in the injection impedance plane. In [10] it was mentioned that the boundary appears as a circle with the following characteristics:

- With radius $r=Z_{t h} /(2 \sin \phi)$ 


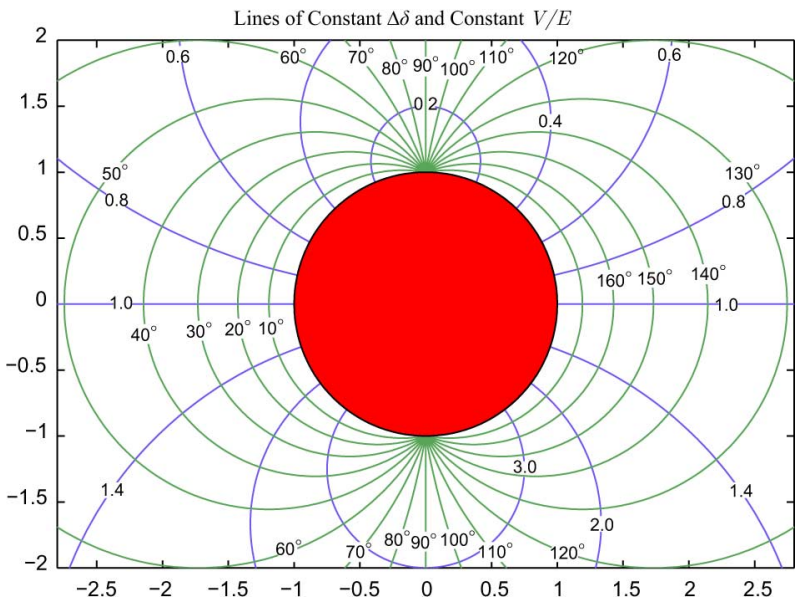

Fig. 11. Illustration of the normalized injection impedance plane when (20) is used. The boundaries appear as a unit circle at the origin. Lines of constant voltage ratio $V / E$ (blue lines) and of constant phase angle difference $\Delta \delta$ from the boundary (green lines) are depicted.

- The center of the circle is located on the imaginary axis where $X_{i n j}=-r$

In the following, the stability boundaries will be normalized in such a way that they appear as a unit circle centered at the origin of the normalized impedance plane. For this purpose, all impedance values $\bar{Z}_{i n j, \star}$ have to be shifted by $r$ in the direction of the imaginary axis and scaled down by factor $r$. To achieve this, the expression (19) is manipulated:

$$
\bar{Z}_{i n j, \star, p u}=\frac{\bar{Z}_{i n j, \star}}{r}+j=\frac{2 \bar{Z}_{i n j, \star}}{Z_{t h, 0}}+j .
$$

By applying (20), the characteristic lines for constant $\delta$ and $V$ and the stability boundary can be used to visualize all of the $k$ operating points $\left\{\bar{Z}_{i n j, i}, \bar{Z}_{t h, i}\right\}$ in the same impedance plane, where the same boundary applies to all operating points.

In Fig. 11, the appearance of the stability boundary and a few lines of constant phase angle margin $\Delta \delta$ and normalized voltage magnitude $V / E$ are visualized in the normalized injection impedance plane. An arbitrary operating point $\left\{\bar{Z}_{i n j, i}, \bar{Z}_{t h, i}\right\}$ can be mapped into this plane by applying (20).

\section{Simulation Results and Large Scale Test}

The following reports on two test-case scenarios, case A and $B$ respectively. In case A, a trip of a single line eventually causes a manually excited machine to cross the boundaries for aperiodic small signal rotor angle stability leading to a collapse in voltage magnitude. In case B, a scenario where long term voltage instability mechanisms eventually cause one of the machines to cross the stability boundary, also leading to a sudden collapse in the system voltages.

\section{A. Test System Description}

In order to validate the method's performance, a time-domain simulation of an instability scenario was carried out on the Nordic32 system reported in [13]. One-line diagram of this system is shown in Fig. 12. The system topology was modified so that the loads in the southern part of the system (buses 1041 to 1045) were supplied through ULTC-transformers. Hence, buses 141-145 where added to the model.

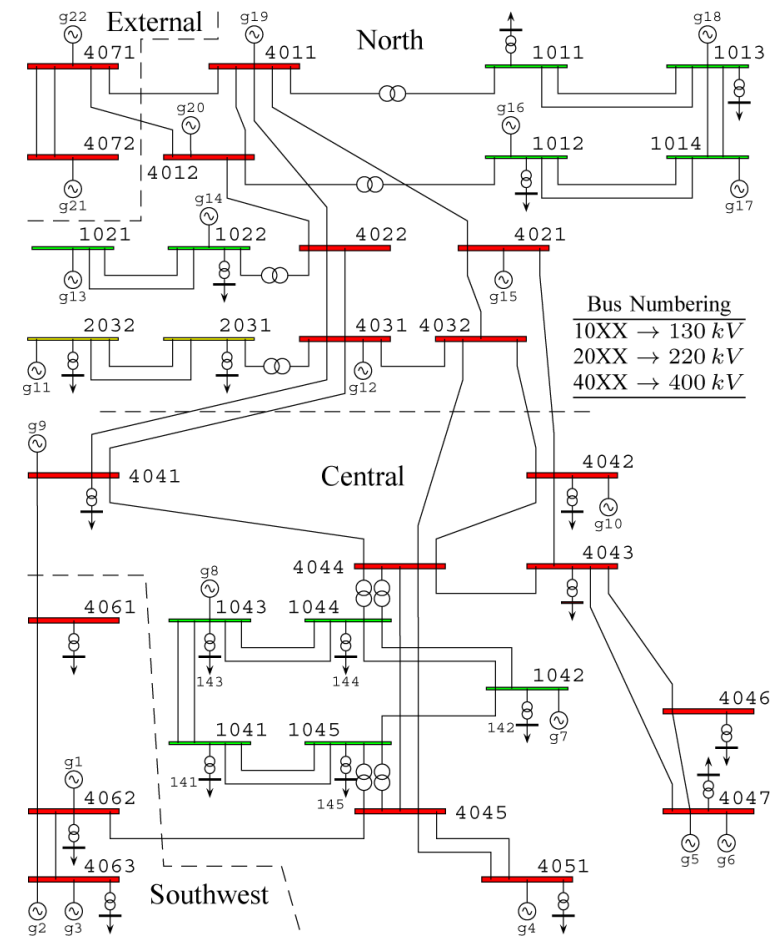

Fig. 12. One-line diagram of the Nordic32 system.

TABLE I

Overview of the INITIAL CONDITIONS USED IN CASES A AND B

\begin{tabular}{|c|l|}
\hline Case & Modification of the initial conditions presented in [13] \\
\hline A & $\begin{array}{l}\text { Generator } g_{13} \text { is put out of order and its load taken over by } \\
g_{11}, g_{19}, g_{21} \text { and } g_{22} \cdot g_{11} \text { is manually excited and one of lines } \\
\text { between } 2031-2032 \text { is disconnected. }\end{array}$ \\
\hline B & Load increase of $190 M W$ distributed over busses $141-145$ \\
\hline
\end{tabular}

The initial conditions used in the two test case scenarios were slightly modified compared with the initial condition presented in [13]. The modifications for both cases are listed in Table I. The initial conditions in both cases represent a very stressed system operation where the excitation voltage of several generators is close to the limits for their OEL.

\section{B. Simulation and Test Results}

1) Case A-Instability of a Manually Excited Machine: To provoke instability, the line between 4021 and 4042 was tripped at $t=5 \mathrm{~s}$. Fig. 13(a) shows the voltage magnitudes on five buses in the system. The disturbance caused powerful but damped electromechanical oscillation, followed by activation of several OELs before a sharp collapse in voltage magnitude was noticed almost $55 \mathrm{~s}$ after the disturbance. The OELs acted successively, starting with $g_{12}$ at $18.4 \mathrm{~s}, g_{13}$ at $23.8 \mathrm{~s}, g_{10}$ at $33.3 \mathrm{~s}$, $g_{15}$ at $42.7 \mathrm{~s}, g_{9}$ at $47.0 \mathrm{~s}$, and finally $g_{8}$ at $47.5 \mathrm{~s}$. The simulation results were used to generate PMU snapshots of the system conditions, which were then used as inputs to the assessment methods. Fig. 13(b)-(f) visualizes the system conditions with respect to aperiodic small signal rotor angle stability at five different instances of time [snapshots I-V in Fig. 13(a)]. The plots depict several operating points that have been normalized by deploying (19) and (20). The round operating points represent the actual operating point of a given machine, while the squared 


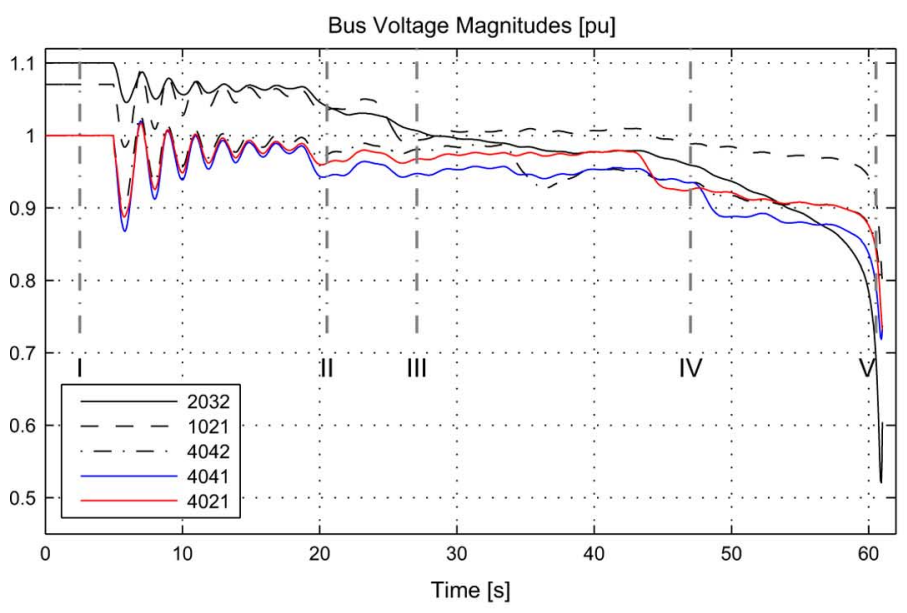

(a)

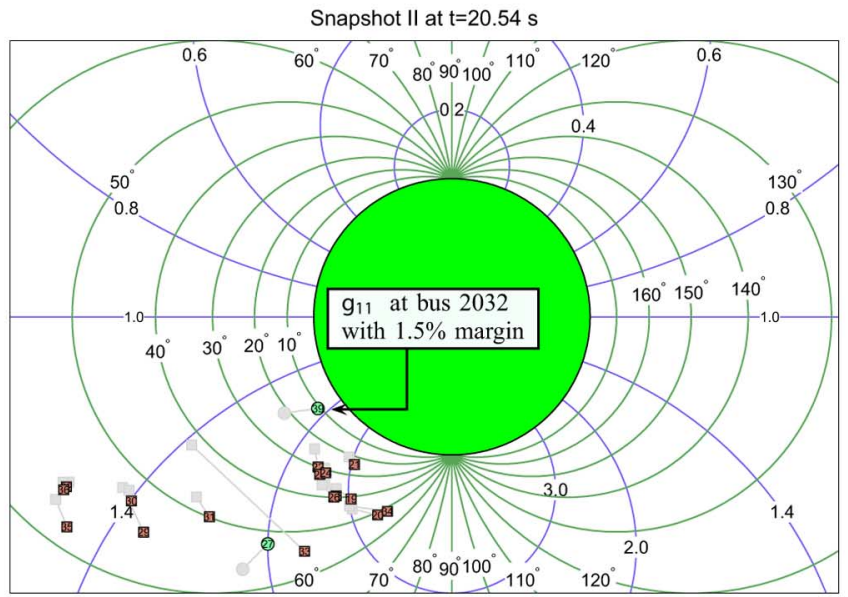

(c)

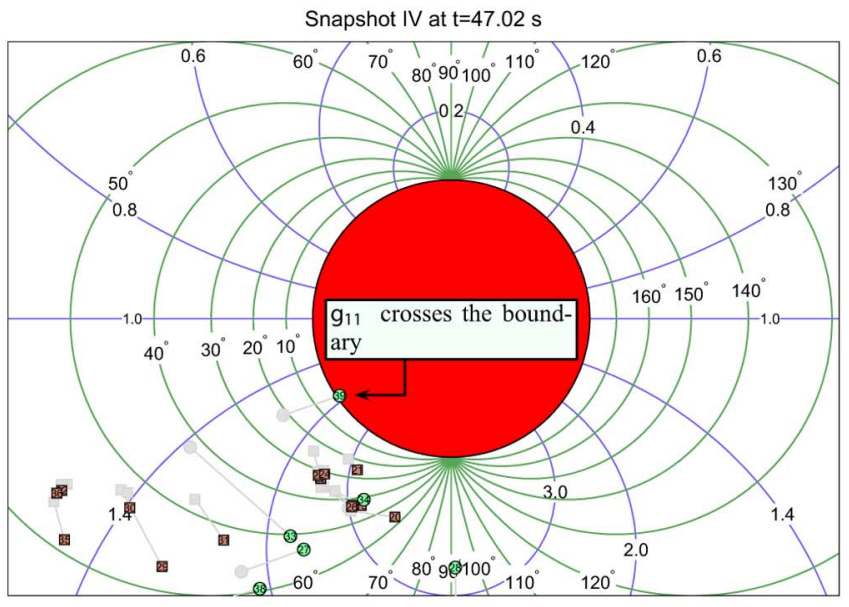

(e)

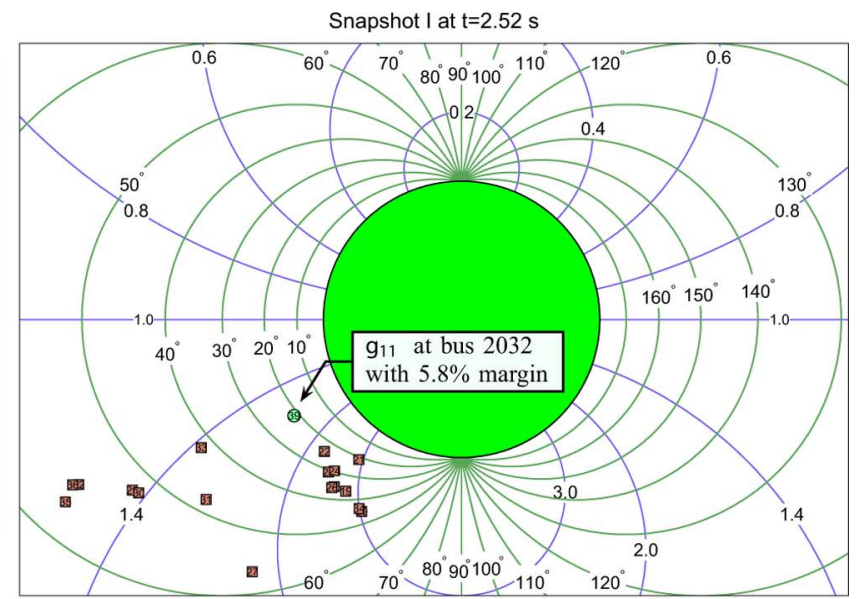

(b)

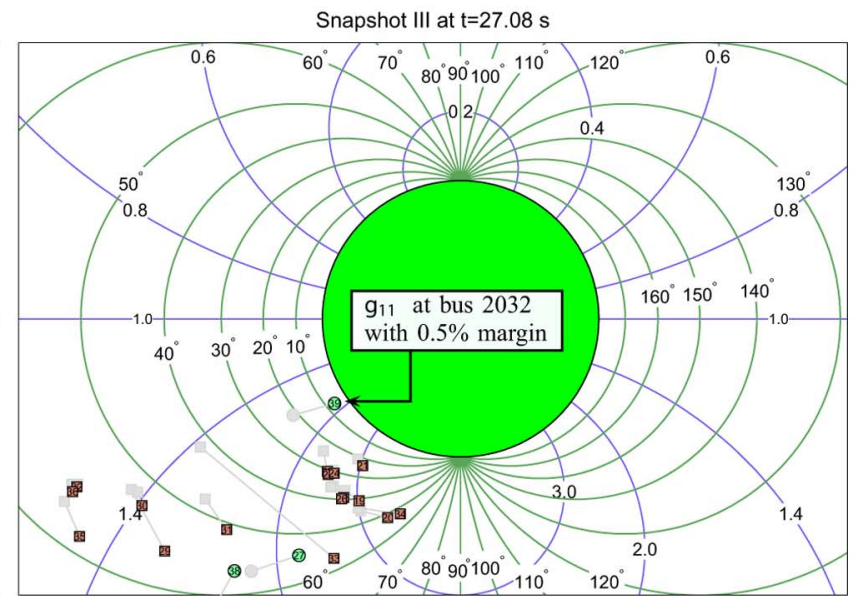

(d)

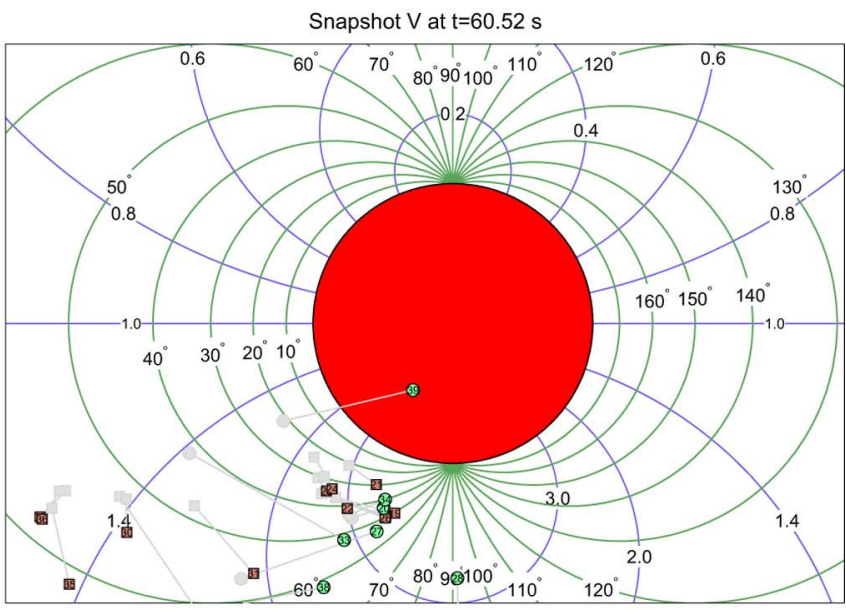

(f)

Fig. 13. Results from test case A. In a) bus voltages at selected buses. In b)-f) snapshots I-V in a normalized impedance plane.

operating points represent the operating point of a given machine if the machine loses its capability to maintain a constant terminal voltage (e.g., when an OEL has been activated). Thus, the squared point does not represent an actual operating point of a machine, but rather a what-if operating point (or a $N-1$ operating point).

Snapshot I in Fig. 13(b) shows the pre-fault conditions, where the operating point for $g_{11}$ is the only active operating point shown in the plot (a manually excited machine) with a 5.8\% ac- tive power margin to the boundary. Snapshot II is taken after the disturbance has been applied and when the OEL of $g_{12}$ is active, hence an additional active operating point appears in Fig. 13(c). In snapshot III, the OEL of $g_{13}$ has also been activated, causing the operating point of $G_{11}$ to move closer to the boundary, resulting in a $0.5 \%$ margin to the boundary.

Snapshot IV in Fig. 13(e) is at the time of boundary crossover of $g_{11}$. After the crossover, $g_{11}$ slowly drifted away from the remaining generators which finally resulted in an abrupt collapse 


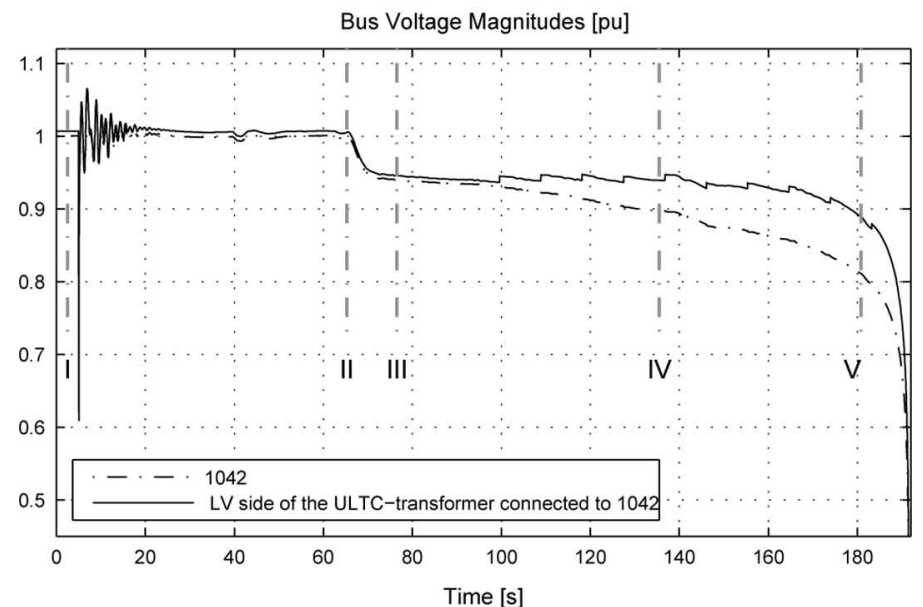

(a)

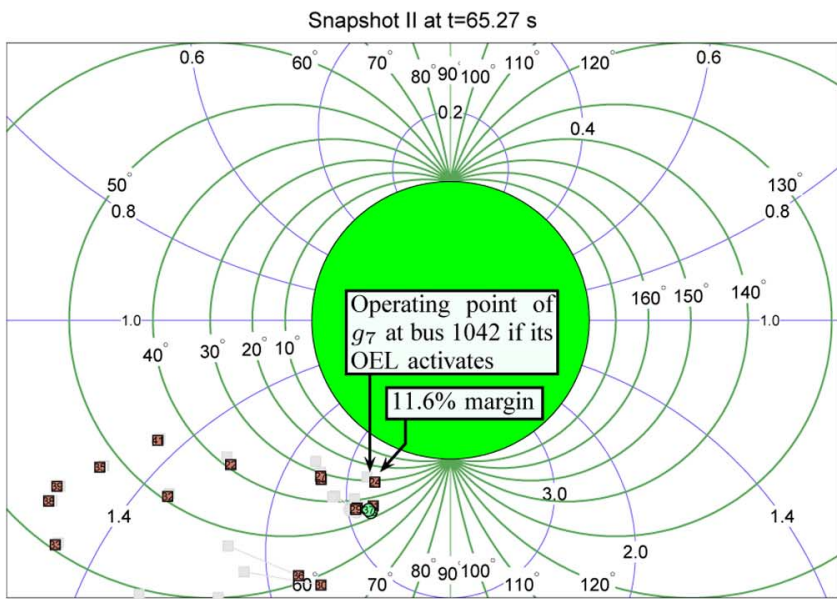

(c)

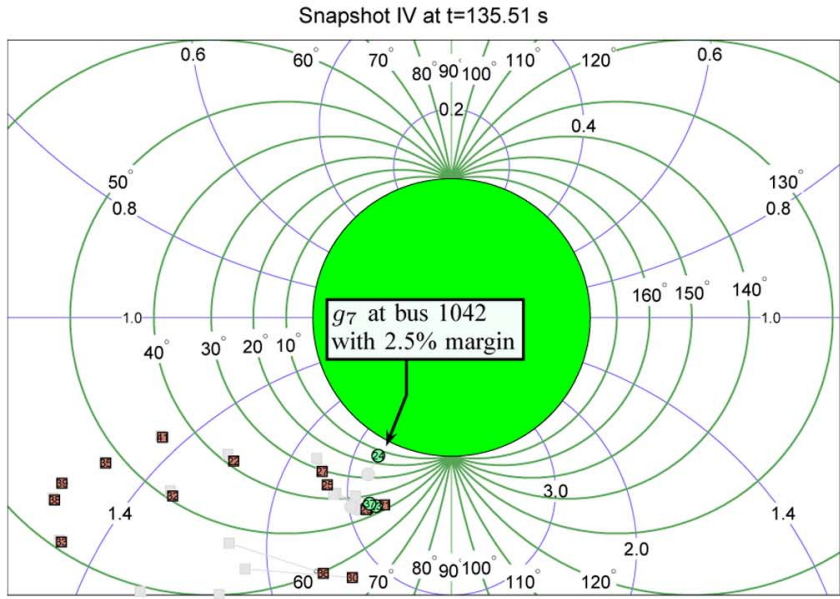

(e)

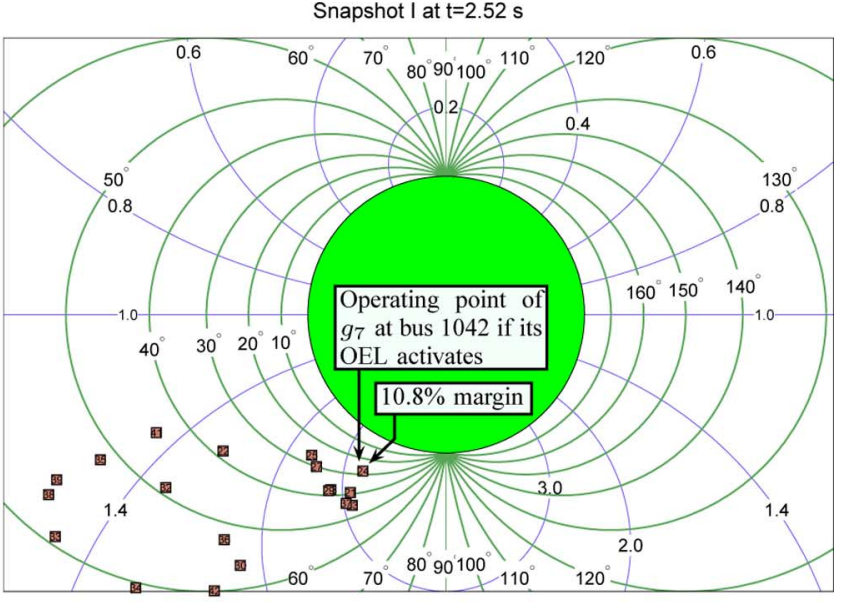

(b)

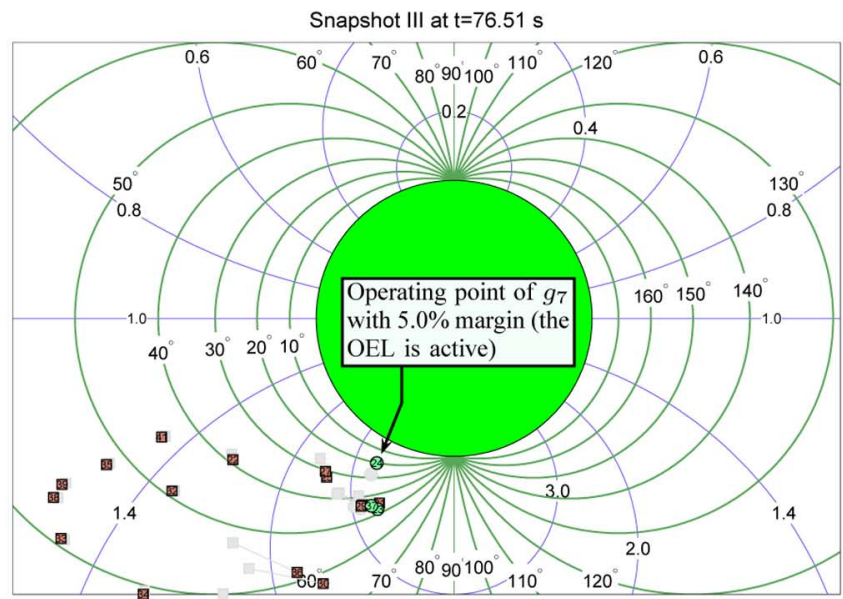

(d)

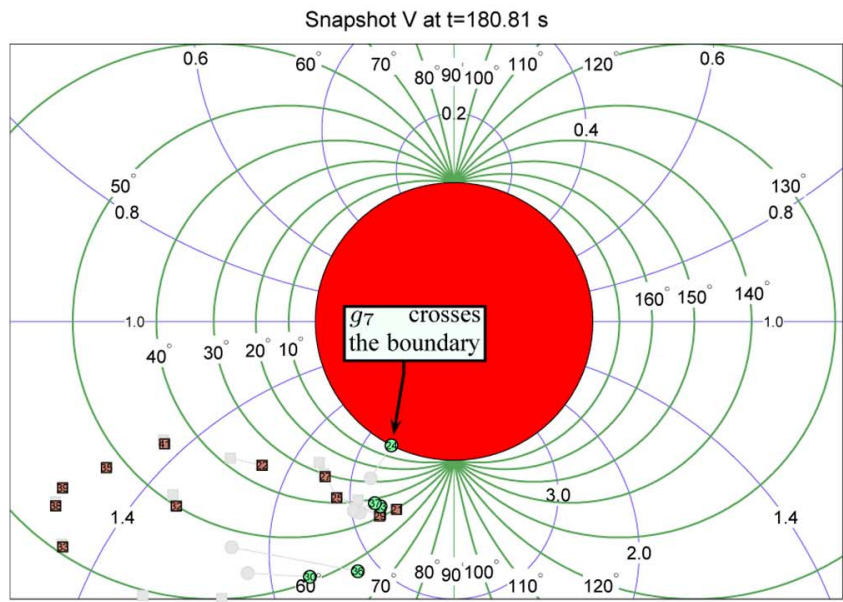

(f)

Fig. 14. Results from test case B (a) voltages on HV and LV side of a ULTC-transformer on bus 1042 (b)-(f) snapshots I-V in the normalized injection impedance plane. The activation of the OEL of $g_{7}$ causes decrease in voltage at bus 1042 and the subsequent ULTC actions move $g_{7}$ closer to its boundary with every tap action (d-e). After the boundary crossover (f), the machine loses synchronism which explains the sudden collapse in voltage magnitude.

approximately $12 \mathrm{~s}$ after the crossover. Snapshot V shows when the voltages in Fig. 13(a) are collapsing.

Case A illustrated how cascading activation of OELs caused the generator $g_{11}$ to gradually drift towards the stability boundary and finally cross the boundary, resulting in a loss of synchronism and the abrupt collapse of voltages in Fig. 13(a).
2) Case B-Voltage Instability Mechanism Causing Instability of a Machine: A voltage instability was caused by applying a short circuit on the line between 4044 and 4032 . The fault was isolated by disconnection of the line $100 \mathrm{~ms}$ after the short circuit. The time response following the disturbance is depicted in Fig. 14(a). The plot shows the voltage magnitudes on 
the low and the high voltage side of the ULTC-transformer on bus 1042 . From the plot, a voltage instability becomes visually apparent following a tap-change at $t=155 \mathrm{~s}$, as the resulting voltage magnitude on the LV-side becomes lower than it was before the tap-change occurred.

In the period between the disturbance and until the sharp collapse in voltage was observed, six OELs acted successively, starting with $g_{10}$ at $39.5 \mathrm{~s}, g_{9}$ at $62.3 \mathrm{~s}, g_{8}$ at $62.8 \mathrm{~s}, g_{7}$ at $65.4 \mathrm{~s}$, $g_{12}$ at $139.4 \mathrm{~s}$, and finally $g_{15}$ at $180.9 \mathrm{~s}$. Generator $g_{7}$ crossed the stability boundary at $t=180.8$, which led to a loss of synchronous operation and eventually a sharp collapse in voltage, as depicted in Fig. 14(a).

In the period from the activation of the OEL at $g_{12}(t=$ $139.4 \mathrm{~s})$ and until the boundary crossover of $g_{7}(\mathrm{t}=180.8 \mathrm{~s})$, the actions of the ULTC-transformers at buses 1041-1045 gradually moved the operating point of $g_{7}$ closer to the boundaries. Bus 1042 became voltage unstable around $t=155 \mathrm{~s}$, resulting in a drift of the operating point towards the boundaries by each tap-changing action of the transformer at bus 1042. Finally, $g_{7}$ crossed the boundary and the activation of the OEL of $g_{15}$ $(180.9 \mathrm{~s})$ further accelerated the instability process.

Plots b-f (snapshots I-V) in Fig. 14 reveal how $g_{7}$ gradually lost synchronism. Prior the disturbance (snapshot I), all machines were operating below the limits set for maximum allowable continuous field current, hence with large margins to boundary. Snapshots II and III are taken before and after the OEL of machine $g_{7}$ becomes active. The reduction of the field current in $g_{7}$ due to the OEL activation caused the operating point to move towards the boundary, resulting in a $5 \%$ stability margin. Snapshot IV shows the situation where the actions of ULTC-transformers had gradually moved the operating point of $g_{7}$ closer to the boundary, resulting in a $2.5 \%$ margin. In the period between snapshots IV and V, the actions of the ULTC-transformers and the activation of the OEL of $g_{12}$ led to a voltage instability at bus 1042 and the subsequent boundary crossover of $g_{7}$ as depicted in snapshot V.

\section{Instability Prevention}

This section illustrates how the assessment method can support the selection of a preventive counter action to avoid the emerging instability. The stability boundary represents the maximum steady state power that a given machine can inject into the grid. Hence, a reduction of the power production from a critical machine is an obvious choice for a counter action that moves the operating point away from the boundaries.

Fig. 15 shows the results from a test were a corrective action is applied to prevent instability in case scenario $\mathrm{A}$, presented in Section V-B1. The counter action is applied at $t=40$, where the percentage active power margin of the critical generator $g_{11}$ is $0.1 \%$. The applied counter action consisted of lowering the set point for the applied mechanical power to $g_{11}$ by $30 \mathrm{MW}$.

Fig. 15(a) compares the voltage magnitudes in the cases where the counter action is applied and where no counter action is applied. It can be seen that reducing the applied mechanical power to $g_{11}$ eventually resulted in stable operation. Fig. 15(b) shows the snapshot at $t=99.86 \mathrm{~s}$ where it can be seen that the operating point has moved away from the boundary, with a percentage active power margin of $0.7 \%$.

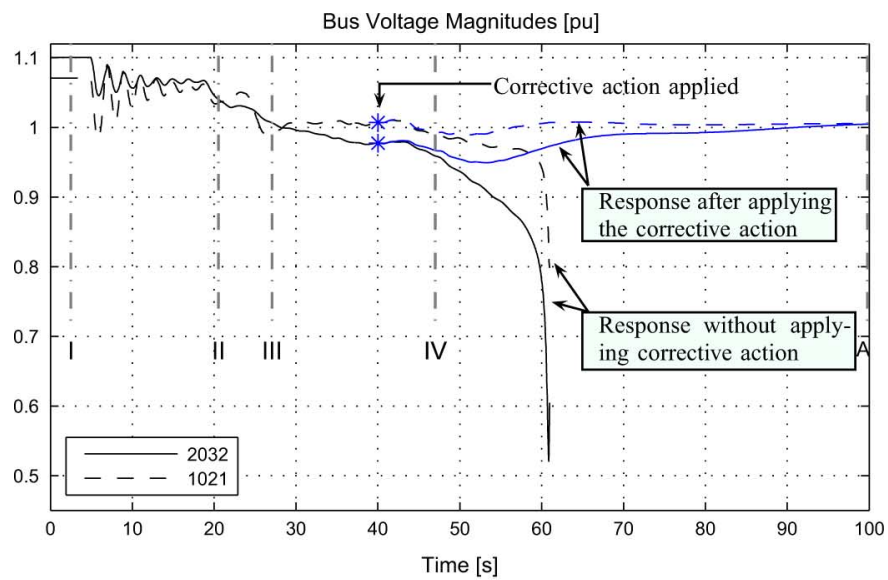

(a)

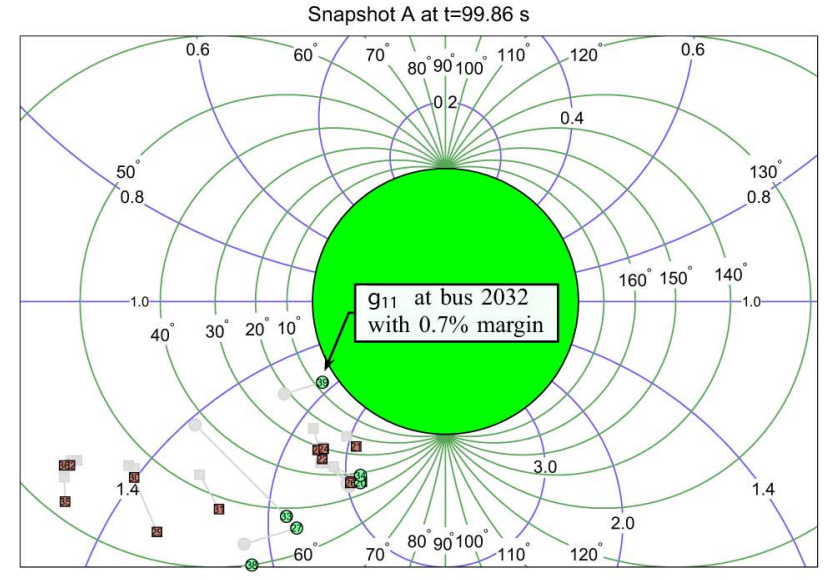

(b)

Fig. 15. Results from a test when a corrective action is taken in case A. In a) voltages at buses 2032 and 1021, where the black lines are the original response and the blue lines show the response when the corrective action has been applied. In b) the snapshot A in a normalized impedance plane.

\section{DISCUSSION}

A new method for assessment of aperiodic small signal stability was presented. The assessment is based on algebraically derived expression for the stability boundary resulting in that only the Thevenin impedance $Z_{t h}$ and the injection impedance $Z_{i n j}$, seen from a generator's node of injection, are needed for the assessment. Resent research shows that the information needed to assess the stability of all the generators in large systems can be obtained in few milliseconds, which makes the presented method well suited for assessment in real-time.

The method's capability of accurately determining when the stability boundary is crossed was demonstrated in Section III. The detailed study of the initial conditions showed that the system regains a stable equilibrium after the first disturbance with a very small margin to the boundary. The test results were in line with these observations, the generator preserved its stability after the first disturbance, but operating very close to the boundary and after a very small second disturbance, the method accurately detected the boundary crossover.

The expressions derived for the mapping of an arbitrary operating point of a given machine into a normalized injection 
impedance plane provide useful means for operator decision support. By expressing the stability margin of a given machine in terms of active power, provides a "distance-to-instability" information in terms of system variables that are meaningful to a system operator and can be used as an early warning indicator for the occurrence of an emerging stability problem.

The two Nordic32 examples provided illustrate the need for developing different types of assessment methods if power system stability is to be assessed in real-time. For case A, the presented method identifies the root cause of the impending instability in good time, while in case B, voltage instability is the underlying mechanism that moves critical machines gradually closer to their boundaries as time evolves. For case B, the presented method can identify and monitor stability margins of the critical machines that eventually will lose synchronism if the voltage instability process continues. To efficiently avoid instability in case B, voltage stability assessment methods are needed which can identify the location of the voltage instability problem and suggest counteractions that effectively stop the gradual degradation of the system conditions. Such an element-wise-assessment of a particular mechanism of stability has the advantage that it provides information about where in the system the problem is emerging and what is the physical nature of the problem. The analytically derived stability boundaries ensure that method can provide a measure of the distance to instability. The where, which type and how close information provides a very useful basis for the development of methods that can automatically determine appropriate counteractions to avoid impending instability and eventual system blackout as was illustrated by a simple example.

\section{REFERENCES}

[1] A. Phadke et al., "A new measurement technique for tracking voltage phasors, local system frequency, and rate of change of frequency," IEEE Trans. Power App. Syst, vol. PAS-102, no. 5, pp. 1025-1038, 1983.

[2] S. Skok et al., "Applications based on PMU technology for improved power system utilization," in Proc. IEEE Power Eng. Soc. General Meeting, 2007, pp. 1-8.

[3] D. Novosel et al., "Dawn of the grid synchronization," IEEE Power Energy Mag., vol. 6, no. 1, pp. 49-60, 2008.

[4] A. Phadke et al., "The wide world of wide-area measurement," IEEE Power Energy Mag., vol. 6, no. 5, pp. 52-65, 2008.
[5] M. Glavic and T. V. Cutsem, "Wide-area detection of voltage instability from synchronized phasor measurements. Part I: principle," IEEE Trans. Power Syst., vol. 24, no. 3, pp. 1408-1416, Aug. 2009.

[6] M. Glavic and T. V. Cutsem, "Wide-area detection of voltage instability from synchronized phasor measurements. Part II: Simulation results," IEEE Trans. Power Syst., vol. 24, no. 3, pp. 1417-1425, Aug. 2009.

[7] H. Jóhannsson, "Development of early warning methods for electric power systems," Ph.D. dissertation, Tech. Univ. Denmark, Kgs. Lyngby, Denmark, 2011.

[8] H. Jóhannsson et al., "Real-time stability assessment based on synchrophasors," in Proc. IEEE Trondheim PowerTech, 2011.

[9] H. Jóhannsson, "Assessment of Power Systems," Patent No. 111681113.6 - 2207, 2011.

[10] H. Jóhannsson et al., "Identification of critical transmission limits in injection impedance plane," Int. J. Elect. Power, vol. 43, no. 1, 2012.

[11] S. Sommer and H. Jóhannsson, "Real-time Thevenin impedance computations," in Proc. 2013 IEEE ISGT Washington Conf., Feb. 2013.

[12] V. Ajjarapu and C. Christy, "The continuation power flow: a tool for steady state voltage stability analysis," IEEE Trans. Power Syst., vol. 7, no. 1, pp. 416-423, Feb. 1992.

[13] M. Stubbe, "Long-term dynamics-phase II (report of CIGRE Task Force 38.02.08)," IEEE Trans. Power Syst., 1993.

Hjörtur Jóhannsson (M'11) received the M.Sc. and Ph.D. degrees in electrical engineering from the Technical University of Denmark, Kgs. Lyngby, Denmark, in 2007 and 2011, respectively.

$\mathrm{He}$ is an Assistant Professor at the Center of Electric Power and Energy (CEE), Department of Electrical Engineering, Technical University of Denmark. His research interests concern the development of methods for real-time stability and security assessment of electric power systems.

Arne Hejde Nielsen (SM'08) is an Associate Professor at the Centre of Electric Power and Energy (CEE), Department of Electrical Engineering, Technical University of Denmark, Kgs. Lyngby, Denmark. He has 30 years' experience in electric power engineering; the first years from ASEA AB, Central Research and Development Department, Sweden, with focus on measurement technology and motor design and control. Over the past decade his focus has been on implementation of renewable energy sources in the power system.

Jacob Østergaard (M'95-SM'09) is a Professor and Head of the Centre for Electric Power and Energy (CEE), Department of Electrical Engineering, Technical University of Denmark, Kgs. Lyngby, Denmark. His research interests include integration of renewable energy, control architecture for future power system, and demand side.

Prof. Østergaard is serving in several professional organizations including the EU SmartGrids advisory council. 